\title{
Income Inequality and Health Disparities in China's Urban and Rural
}

\author{
Tingyu He
}

\author{
Chengdu Foreign Languages School, Chengdu, Sichuan, 610031, China
}

Corresponding author.Email: 1634453517@qq.com

\begin{abstract}
In the past 4 decades, along with rapid economic development, social gradients in both health and social-economic status (SES) have come into picture in China. Associated with SES, health inequality has become a severe issue of the nation, at whose root lies a key factor: income. The author briefly reviews some published research and studies on income inequalities and its impact on health status across the country, and suggests that spatial disparities of economic development among and within provinces largely generate health inequality in China. With farther elaboration, the author focused on three dimensions: 1. the urban-rural gap in China, 2. health status of the elderly, and 3. health status of children, as representatives to illustrate the association. According to published research work, income differences have resulted in health disparities at both an individual level and a regional one. That is, not only that income inequality exists among individuals in China, but it also has a clear spatial pattern. The author concludes that income gradients are positively connected to health status of both the elderly and children in China, and health policymakers must pay attention to these two subgroups and offer them more approachable health services.
\end{abstract}

Keywords: Income, inequality, health, disparities, China

\section{INTRODUCTION}

The relationship between incomes and health has been seriously considered since the 1980s [1]. For instance, Haitian men could only live in full health for 27.8 years in 2010, while healthy life expectancy of Japanese male was 70.6 the same year [2]. In addition, the difference of death rate between individuals from the poorest quintile of families and the wealthiest ones is up to $86 \%$, with influential factors adjusted. [3] For a given society, health may be associated with the gradients of incomes within [4]. Nowadays people have a simple consensus that the effects of income inequality on health is just like the "Wilkinson hypothesis" (increased income inequality in a society is related to worse health performance) [5]. Public goods providers have long devoted to eliminate health inequalities, but those are strongly associated with other social factors such as socioeconomic status (SES), public education, etc. [6]. In China, with huge SES differences between east and west, rural and urban areas, health care systems committed to improve overall health are still facing challenges. Many researchers have pointed out that the impact of different measurements of SES reflects in incidence of diseases in China, such as stroke, demonstrating that inequalities in the risk of stroke was severe for people who lived in rural areas. Income, as a factor associated with SES, plays a very important role in the situation. Since the 1980 s, a rapid growth in economy was greeted in China due to the transition to a market economy. Along with which, however, came a constantly growing gap between income groups [7] that had soon become a factor strongly generating health status of the Chinese population. Moreover, certain studies further indicate that negatively affected health situation will in turn bring a even larger gap of income inequality, and hence, create a vicious cycle [8].

In this paper, the following questions will be investigated: the urban-rural gap of income in China, as well as the impact of income inequality on the elderly and children.

\section{THE EMBODIMENT OF URBAN-RURAL GAP IN CHINA}

Since 1980s, China has made great progress in healthcare system and has brought better health outcomes parallel with its amazing economic development. For example, the life expectancy at birth was 74.8 years in 2010 which only was 67.9 years in 
1981 [9]. Even so, there have long way to realize equilibrium in both health status and health services through the entire country, especially within and between urban and rural residents. It is a general phenomenon that the rural population has less access to health resources such as insurances. And more seriously, the gaps of income inequality have widened constantly since the economic transition, due to urban-rural dualistic economic structure [10] that mean there had 2 health systems worked at the same time, for urban areas and rural areas respectively. In rural areas, the economical growth depended on single industry deeply, such as agriculture and breeding industry, which can't support the rapid rising need of health care service. Meanwhile, the economic boom in urban areas make health care service have tilted to in reverse situation, that is, receiving more healthcare resources with a higher quality, and hence creating disparities of healthcare between urban and rural.

As mentioned above, there are two healthcare systems working simultaneously, leading to different health insurance schemes in China's urban and rural. Before 1980s, Chinese farmers had rural cooperative medical system which meet the need of basic health care of them. But the system has breakdown in most of the rural areas after social reform and opening up policy started, most Chinese farmers had lost protection of health care insurance. As we see, the huge differences of basic medical insurance scheme for rural farmers and urban employee, only $9.5 \%$ of farmers had been covered by insurance in 1998 compared that almost all urban formal employees had been covered at the same year. But it must been noted that there still had difference within urban residents, unemployed urban residents and migrant workers had low covered ratio of health care insurance just like rural farmers. The growing inequality of health had drawn a lot of criticism, not only the inequality between rural and urban areas, but more important was that the growth of health investment had not caught the step of economical growth, thus, only $13 \%$ of rural people and $22 \%$ of urban people were insured in 2003 [11]. Therefore, Chinese government restarted the New Cooperative Medical Scheme (NCMS) for rural people in 2002, and new basic medical insurance schemes for vulnerable groups in urban areas such as the unemployed, students and the elderly in 2007. However, there still had financial unbalance problem between NCMS and the urban health insurance net. Specifically, in 2012 the NCMS received a fund of 248.34 billion CNY, while urban insurance schemes had 553.92 billion CNY of funds [12].

According to the discuss above of embodiment of urban-rural health inequality in China, we found that the fundamental reason of health inequality generally associated to disproportion of economical growth. And other factors, such as insurance scheme, the investment for health care by government, have also contributed to the issue.

\subsection{The influence of income inequality on urban and rural}

Bin Guo et al. investigated the inequality in health services in China, focused on within and between rural and urban [13]. The study identified the socioeconomic factors involved the inequalities. The study has call in 177,501 questionnaires in the 2008 National Health Services Survey (NHSS), include 56,456 families throughout 31 provinces, 128,753 families in rural areas and 45,645 in urban. They have found that: (a) urban residents have longer expectation of life and better education backgrounds. (b) urban residents are more likely to have poorer health condition with chronic diseases, compared to rural residents, but a more favorable health insurance coverage. (c) The need of inpatient services is more urgent in in rural areas with lower income. (d) In many cases, household income is a decisive factor for utilizing outpatients or inpatient services.

In another study, the researchers hope to reveal that health inequality between rural and urban area in China by the index out-of-pocket (OOP) [14]. They focused on 37,605 Chinese residents from 11,409 families living in different areas in 2011 and 2012 [14]. The basic situation includes education, age, and financial condition, and their findings are as follows: (a) The ratio of urban residents with college education completed was 10 times as high as the ratio of rural population $(25 \%$ vs. $2 \%$ ). (b) The population of residents over 60 years old was larger in urban areas (14.3\% vs. $13.1 \%)$. In contrast, population of children under 14 years old was smaller in urban areas, but there was a decline of this population observed in rural(19.6\% in 2011 vs. $18.6 \%$ in 2012). (c) The OOP appeared to be lower for urban residents than rural ones in terms of their total consumption. (d) Non-food consumption expenditure (NFCE) was lower in urban than in rural areas. In summary, the index of both OOP and NFCE indicated a better living condition in urban areas in China [14].

In conclusion, although there has been a rapid economic development in China in the past 40 years, there is still noteworthy income inequality which leads to inter-regional health inequality in China, especially between urban and rural, that can be explained by social-economic differences caused by China's urban-rural dualistic economic structure and its vast territory. This results in extensive inequalities in work opportunity, salary level, education, population quality, distribution of social welfare, and most importantly, heath. 


\subsection{Health disparities of the elderly in urban and rural areas}

As well as many other developed countries, China is also facing the problem of aging population attributed to declining birth rate and increasing life expectancy. By 2017, the number of older people aged 60 and above had reached 240.90 million, making up $17.3 \%$ of Chinese population. Furthermore, as predicted, by the year of 2050 , at least $30 \%$ of the population will be constituted by people over 60 years old [15]. Statistically speaking, a person's health care expenditures over the age of 65 accounts for approximately $70 \%$ of his life time health care expenditures, treating hypertension, heart disease, stroke, diabetes, Alzheimer's, and severe depression because of the decline of physical functions and loneliness [16].

A study of which data derived from a repeated cross-sectional survey was entitled by Guangzhou Community Health Survey (GCHS 2013) [17]. There were 17,290 individuals included in this study. The main findings of the study were as follows: (a) healthcare professionals with adequate health literacy and HISBs is distributed with a clear pro-rich pattern(HISB refers to the ways in which individuals seek information regarding their health, such as risks, illnesses, and health-protective behaviors to improve their health) from healthcare professionals; (b) this pattern is caused mainly by educational background; and (c) income inequalities is a factor primarily generating the urban population in terms of HISBs.

Chenjing Fan et al published a paper in August 2019 aiming to learn the living conditions of the elderly living in different regions in China and assessed some factors which probably play a role in the conditions, such as their socio-economic, psychological and physical health status [18]. The data came from the fourth sampling survey of the elderly population (4th SSEP) in 2015, which was the first research of 1\% sampling survey of the elderly (over 60 years old) in China, carried out by The China Research Center on Aging (CRCA), in which 222,179 people over 60 years old had participated. The general trend of the results had been described as "high in coastal, poor in central" spatial pattern by researchers, the wealthiest coastal provinces such as, Jiangsu, Shanghai, and Zhejiang had the highest personal income and education level and the best medical care, forming a top level health region around the Yangtze River Delta. The fact revealed health inequality has geographical inhomogeneity, and it also applies to the elderly population in wealthier regions, for they tend to have more access to better healthcare services. However, this spatial pattern far from a critical factor, for inter-provincial differences can only account for about $2.4 \%$ of elderly health differences, while the rest are closely related to factors such as gender, marital status, social interaction and frequency of exercise, which have more to do with individual economic states, which further proves that income is a crucial factor of determined elderly health inequality. In addition, the place of residence also contributes to elderly health. Elderly people living in urban areas had better health status than those living in rural or suburb. This may reflect that urban-rural gap of health inequality in elderly as mentioned initial.

\subsection{Health disparities of children in urban and rural areas}

Much of existing literature studying areas with different levels of development in China have demonstrated that children's health is positively associated with household incomes. Shen et al. reviewed that the difference of height between urban and rural children has widened since the 1980s along with China's Reform and Opening Policy [19]. Lu Chen et al. also examined the health of children with the China Nutrition and Health Survey (CHNS) datafrom 1989 to 2009as representatives of the era of a central planning economy system and the era of a market one [20]. The results showed that the average health status of children have improved in tin this period of time. Two possible explanation were concluded in this study. First, food diversity and high protein food consumption gradually came into picture and had been paid attention to in both urban and rural areas in China. For example, money income soon replaced food rationing and food coupons in the early 1990s to obtain food, and therefore became relevant to child health. Second, healthcare systems in China, especially those for children and maters, were significantly improved.

Furthermore, the results also suggest that the poor had poorer health in China but less access to both health care and education resources. Moreover, at the regional level, provinces with better healthalways appear to have higher rate of middle school enrollments. This suggests that child health is related not only to healthcare, but also to education resources. Therefore, despite the overall improvement in child health, children in rural areas tend to have less resources to healthcare or education, which leads to the constantly growing difference of health status between urban and rural children.

Another article [21] interestingly used the sum of decayed, missing and filled teeth (DMFT) index as a parameter to represent the health inequality. The study observed 772 preschool children from 15 kindergartens distributed in urban and rural areas in northeast China. The researchers found that children's dental caries were negatively but strongly connected to household income and mother's education level, while children's weight and height were positively associated with income. In conclusion, in comparison with children in urban areas, 
rural children were more likely to have dental caries but less likely to grow taller or heavier.

According to the Report on China's Migrant Population Development of 2015, there were 253 million migrants within the country in 2014 [22]; According to the Report on China's Migrant Population Development 2016, released by the Migrant Population Services and Management Division of National Population and Family Planning Commission, $60 \%$ of young and middle-aged migrants in China chose to migrate with their spouses and children in 2015 [23], making migrant children a large population. The researchers compared health status between local and migrant children [24] and revealed that migrant children were significantly less likely to use community-based child healthcare. Furthermore, this study confirmed that families with mothers' education level higher were more likely to offer children the use of physical examination services, but father's educational level and occupation actually have a higher impact on the use of children healthcare compared with these factors among mothers. The author believes that this situation is partially caused by the more superior status held by fathers, due to the traditional belief of paternity. That is, fathers actually get to decide whether their children are receiving regular physical examinations, and fathers with better education and health literacy would naturally provide children with better healthcare.

\section{LIMITATIONS}

The author aims to discuss the issue of income inequality and the impact it has on health status in China. However, because the territory of the country if vast, and levels of economic development and cultural beliefs vary from place to place, many details have not been included in this brief review. Moreover, due to the absence of professionally developed epidemiology research and longitudinal studies, present data are mostly drawn from sampling statistics entitled by governments, and hence may lead to offsets in results.

\section{CONCLUSIONS}

As Chinese economy grows rapidly, economic differences have drawn much attention, causing income disparities, affecting people in different areas and regions. According to published research work, income differences have resulted in health disparities at both an individual level and a regional one. It is critically important to understand the variation in health across income groups, and in particular to take into account the effects of urban/rural residence on the degree of income-related health inequalities. Furthermore, the income gradients are also affecting different social groups, such as the elderly and children. With China going into grey society rapidly, more attention should be paid to health inequality problems among elderly people. In addition, the situation of health inequality among children is also strongly associated to household incomes and spatial factors, and the problem is particularly severe for migrant children. Therefore, it is suggested that health policymakers should pay more attention to the welfare of the elderly and children, and it is very urgent to implement politic and system reform to provide these groups of population with more public healthy product and services.

\section{REFERENCES}

[1] C.J.L. Murray, E.E.Gakidou, J. Frenk. Critical reflection : health inequalities and social group differences: what should we measure? Bull World Health Organ, 1999(77): 537-544.

[2] J.A. Salomon, H. Wang, M.K. Freeman, T. Vos, A.D. Flaxman, A.D. Lopez, et al. Healthy life expectancy for 187 countries, 1990 2010: a systematic analysis for the Global Burden Disease Study 2010. Lancet, 2012(380): 2144-2162.

[3] S.V. Subramanian, L.K. Ackerson, M. Subramanyam, K. Sivaramakrishnan. Health inequalities in India: the axes of stratification. Brown J World Aff, 2008(14): 127.

[4] M.C. Arcaya, et al. Inequalities in health: definitions, concepts, and theories. Glob Health Action, 2015(8): 27106.

[5] N.Z. Bakkeli. Income inequality and health in China: a panel data analysis. Soc Sci Med. 2016(157): 3947.

[6] P. Townsend, N. Davidson. (Eds.) Inequalities in health: black report. Harmondsworth, Middlesex: Penguin Books Ltd; 1982: 240.

[7] H. Liu, J.X. Chao. Income-Related Health Inequality of Urban Residents in China and Influencing Factors. Chin. Gen. Pract. 2009(12): 1609-1610. (In Chinese)

[8] L. Qi, Z. Li. The income-related mobility of health and health care utilization. Econ Res J. 2011, 46(9): 83-95.

[9] National Bureau of Statistics. People's Republic of China. China Statistical Yearbook 2018. Beijing: China Statistics Press; 2018.

[10] China Health Statistics Yearbook 2013. Beijing: Ministry of Health of China; 2014.

[11] T. Alcorn, B. Bao. China progresses with health reform but challenges remain. Lancet, 2011(377): 1557-1558. 
[12] A. Wagstaff, M. Lindelow, G. Jun, et al. Extending health insurance to the rural population: an impact evaluation of China's new cooperative medical scheme. J Health Econ, 2009(28): 1-9.

[13] B. Guo, X. Xie, Q. Wu, X. Zhang, H. Cheng, S. Tao, H. Quan. Inequality in the health services utilization in rural and urban china: A horizontal inequality analysis. Medicine, 2020(99): 2(e18625).

[14] L. Wang, A. Wang, D. Zhou, G. FitzGerald, D. Ye, Q. Jiang. An Empirical Analysis of Rural \& Urban Differences in Out-Of-Pocket Health Expenditures in a Low-Income Society of China. PLoS ONE, 2016, 11(5): e0154563.

[15] J. Banister, D.E. Bloom, L. Rosenberg. Population aging and economic growth in China. the program on the global demography of aging working paper, 2010, 53.

[16] Y. Zeng, J. Lu, X. Lei, et al. Healthy Aging in China: Trends and Determinants. Beijing: Science Press, 2018.

[17] J. Sun et al. Socioeconomic Inequality in Health Outcomes Among the Elderly: Evidence from a Cross-Sectional Study in China. Risk Management and Healthcare Policy, 2020(13): 397-407.

[18] C. Fan, et al. Elderly Health Inequality in China and its Determinants: A Geographical Perspective.Int. J. Environ. Res. Public Health, 2019(16): 2953.

[19] T. Shen, J.P. Habicht, Y. Chang. Effect of economic reforms on child growth in urban and rural areas of China. N Engl J Med, 1996(335): 400-406.

[20] Chen et al. Income-related children's health inequality and health achievement in China. International Journal for Equity in Health, 2014(13): 102.

[21] A. Shen, et al. The Socioeconomic Inequality in Increment of Caries and Growth among Chinese Children. Int. J. Environ. Res. Public Health, 2020(17): 4234.

[22] Y. Lin, et al. The social income inequality, social integration and health status of internal migrants in China. International Journal for Equity in Health, 2017(16): 139.

[23] Department of Migrant Population, National Health and Family Planning Commission. China's migrant population development report in 2016 (in Chinese).Cited on: 12 Jan 2020. Available from: http://www.nhc.gov.cn/rkjcyjtfzs/pgzdt/201610/5 7cf8a2bbafe4b4d9a7be10d10ae5ecf.shtml.

[24] X. Ke et al. Inequality in health service utilization among migrant and local children: a crosssectional survey of children aged 0-14 years in Shenzhen, China. BMC Public Health, 2020(20): 1668. 